\title{
Control of density fluctuations in atomistic-continuum simulations of dense liquids
}

Kotsalis, E.M.; Walther, Jens Honore; Koumoutsakos, P.

\section{Published in:}

Physical Review E

Link to article, DOI:

10.1103/PhysRevE.76.016709

Publication date:

2007

Document Version

Publisher's PDF, also known as Version of record

Link back to DTU Orbit

Citation (APA):

Kotsalis, E. M., Walther, J. H., \& Koumoutsakos, P. (2007). Control of density fluctuations in atomistic-continuum simulations of dense liquids. Physical Review E, 76(1), 016709. https://doi.org/10.1103/PhysRevE.76.016709

\section{General rights}

Copyright and moral rights for the publications made accessible in the public portal are retained by the authors and/or other copyright owners and it is a condition of accessing publications that users recognise and abide by the legal requirements associated with these rights.

- Users may download and print one copy of any publication from the public portal for the purpose of private study or research.

- You may not further distribute the material or use it for any profit-making activity or commercial gain

- You may freely distribute the URL identifying the publication in the public portal 


\title{
Control of density fluctuations in atomistic-continuum simulations of dense liquids
}

\author{
E. M. Kotsalis, ${ }^{1}$ J. H. Walther, ${ }^{1,2}$ and P. Koumoutsakos ${ }^{1}$ \\ ${ }^{1}$ Computational Science and Engineering Laboratory, ETH Zurich, CH-8092, Switzerland \\ ${ }^{2}$ Department of Mechanical Engineering, Technical University of Denmark, DK-2800 Lyngby, Denmark
}

(Received 30 December 2006; revised manuscript received 21 March 2007; published 30 July 2007)

\begin{abstract}
We present a control algorithm to eliminate spurious density fluctuations associated with the coupling of atomistic and continuum descriptions for dense liquids. A Schwartz domain decomposition algorithm is employed to couple molecular dynamics for the simulation of the atomistic system with a continuum solver for the simulation of the Navier-Stokes equations. The lack of periodic boundary conditions in the molecular dynamics simulations hinders the proper accounting for the virial pressure leading to spurious density fluctuations at the continuum-atomistic interface. An ad hoc boundary force is usually employed to remedy this situation. We propose the calculation of this boundary force using a control algorithm that explicitly cancels the density fluctuations. The results demonstrate that the present approach outperforms state-of-the-art algorithms. The conceptual and algorithmic simplicity of the method makes it suitable for any type of coupling between atomistic and continuum descriptions of dense fluids.
\end{abstract}

DOI: 10.1103/PhysRevE.76.016709

PACS number(s): 47.11.St, 02.70.Ns, 47.11.-j, 02.70.-c

\section{INTRODUCTION}

The modeling and simulation of systems, such as biosensors embedded in aqueous environments [1-4], microfluidic channels with nanopatterned walls, or bluff bodies with superhydrophobic surfaces [5], requires a multiscale approach. In this approach molecular effects such as hydrophobicity need to be properly accounted in the evolution of the continuum system while in turn macroscale effects such as shear forces need to be incorporated in the dynamics of the atomistic system.

The effects of nanoscale physics on macroscale systems is often modeled through the use of appropriate boundary conditions. One example is the no-slip condition for the NavierStokes equations that is widely applied in fluid mechanics problems. A number of experimental and computational studies have demonstrated, however, the shortcomings of this approach for problems such as the moving contact line [6], driven cavity flows [7], and flows in microchannels with nanopatterned walls [8]. In addition a dynamic coupling may be necessary as the macroscale shear forces affect the atomistic flow characteristics [9], while it has been argued in [10] that molecular dynamics simulations alone may not provide suitable boundary conditions to the Navier-Stokes equations at the boundary. Hence, a multiscale modeling approach is necessary in systems with a strong coupling between atomistic and continuum phenomena $[11,12]$.

Hybrid computations have been proposed in order to couple effectively atomistic and continuum descriptions for dense fluids. The atomistic effects are modeled using molecular dynamics (MD) while macroscale phenomena are described by the discretized, incompressible Navier-Stokes equations. Hybrid techniques can be distinguished in the way information is exchanged between the two descriptions. In flux exchange schemes [13-16] the two descriptions communicate at an interface requiring a conservative exchange of fluid properties, while Schwartz domain decompositions $[6,17,18]$ require an overlap region where the atomistic and continuum descriptions coevolve. In both algorithms, a criti- cal issue is the elimination of periodicity from the MD system that is often associated with spurious anisotropies in the particle distributions. Remedies such as repulsive wall potentials $[13,14]$ and buffer regions [15-17] have been proposed in order to circumvent this difficulty. Recently Werder et al. [18] combined a hard wall with boundary potential based on the radial distribution function of the system that is being simulated in order to impose the local system pressure. This scheme was found to significantly reduce the density perturbations in the molecular system compared to existing algorithms. The present study, however, demonstrates that even this approach cannot fully eliminate density oscillations whose amplitude depends on the state point of the liquid.

In this work we extend the hybrid technique presented in [18] and employ a control algorithm to adjust the boundary force in order to eliminate the oscillations in the density when coupling the atomistic to the continuum domains. We note that a similar control approach was proposed by Pivkin and Karniadakis [19] in the context of dissipative particle dynamics. The present control algorithm monitors the gradient of the density oscillations, and it is combined with a specular wall and a particle insertion algorithm that were detailed in [18]. We note that in the present work we do not employ a buffer region [15-17] that is extending outside the active MD domain, thus reducing the computational cost. Buffer regions for flux-based schemes have been shown [15] to guarantee the correct mean and variance of the particle density in the active MD region by allowing the liquid to be in equilibrium with its gas phase at the buffer. In Schwarzdomain decomposition algorithms fluctuations in the density, even at the buffer region, reduce the convergence rate of the algorithm as momentum conservation requires an iterative process [18]. In the present method the length of the overlapbuffer region, in the direction where the periodicity is broken, is independent of the cutoff used in the MD simulation. This is due to the fact that the external boundary force is conservative depending only on the distance and can be viewed as the gradient of a potential. Hence the "buffer" in our method is only restricted to the region where we impose the velocity boundary condition from the continuum whereas 


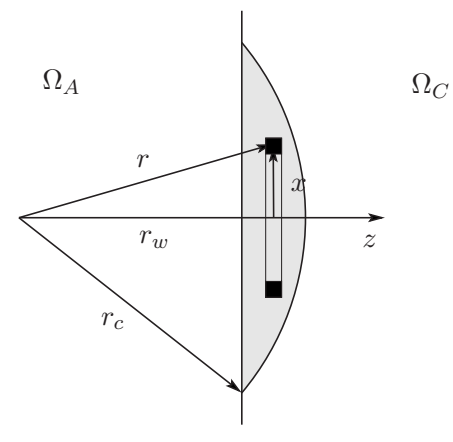

FIG. 1. Integration domains for the effective boundary force [Eq. (2)]. The force contributions along $z$ are integrated over the shaded area. The number of atoms in the infinitesimal ring element is $2 \pi \rho_{n} g(r) x d x d z$, where $\rho_{n}$ is the average number density and $g(r)$ the radial distribution function. $\Omega_{A}$ and $\Omega_{C}$ denote the atomistic and the continuum domains, respectively.

in the case of the flux-based methods this buffer should be large enough so that the atoms and molecules inside the bulk liquid MD region do not interact with the rarefied region. Finally, as demonstrated in $[20,21]$ in flux-based schemes where the pressure tensor is needed, one has either to collect a large amount of samples, thus resulting in a larger computational cost, or use a larger cell size at the cost of having a lower spatial resolution.

\section{METHODOLOGY}

The atomistic region is described by MD simulations subject to nonperiodic boundary conditions (NPBCs). The position $\mathbf{r}_{i}=\left(x_{i}, y_{i}, z_{i}\right)$ and velocities $\mathbf{v}_{i}=\left(u_{i}, v_{i}, w_{i}\right)$ of the $i$ th particle evolve according to Newton's equation of motion:

$$
\begin{gathered}
\frac{d}{d t} \mathbf{r}_{i}=\mathbf{v}_{i}(\mathbf{t}), \\
m_{i} \frac{d}{d t} \mathbf{v}_{i}=\mathbf{F}_{i}=-\sum_{j \neq i} \nabla U\left(r_{i j}\right),
\end{gathered}
$$

where $m_{i}$ is the mass and $\mathbf{F}_{i}$ the force on particle $i$. The interaction potential $U\left(r_{i j}\right)$ models the physics of the system. Here we consider the monoatomic fluid of argon. Thus

$$
U\left(r_{i j}\right)=U_{12-6}\left(r_{i j}\right)+U_{m}\left(r_{w} ; \rho, T\right),
$$

where $U_{12-6}$ is the 12-6 Lennard-Jones $(\mathrm{LJ})$ potential

$$
U_{12-6}\left(r_{i j}\right)=4 \epsilon\left[\left(\frac{\sigma}{r_{i j}}\right)^{12}-\left(\frac{\sigma}{r_{i j}}\right)^{6}\right],
$$

$r_{i j}$ denotes the distance between the $i$ and $j$ atoms, and $\sigma$ and $\epsilon$ are the length and energy scale of the LJ potential (for argon $\epsilon=0.996 \mathrm{~kJ} \mathrm{~mol}^{-1}$ and $\left.\sigma=0.340 \mathrm{~nm}\right)$. The term $U_{m}\left(r_{w} ; \rho, T\right)$ accounts for the interaction of the atomistic region with the surrounding medium. It depends on the distance to the outer boundary of the atomistic domain $r_{w}$, the local density $\rho$, and the local temperature $T$ of the fluid. All interaction potentials are truncated for distances beyond a

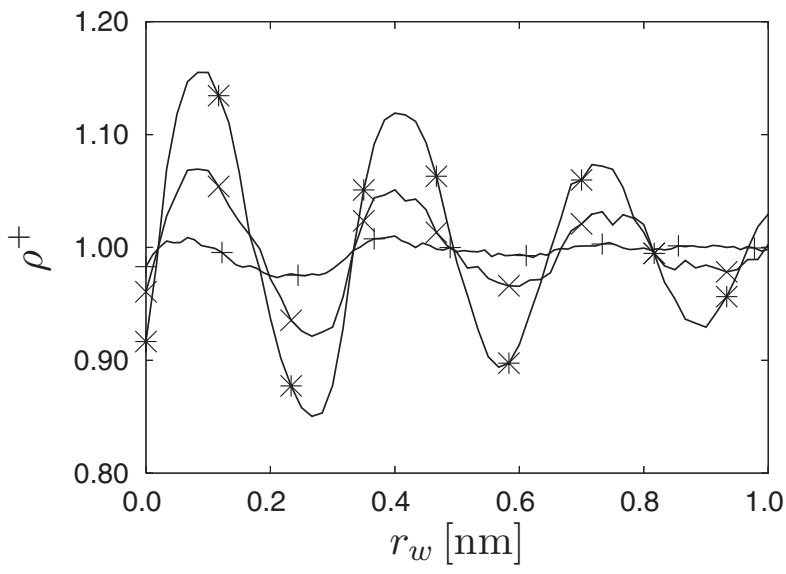

FIG. 2. The reduced density values $\left(\rho^{+}=\rho / \rho_{\text {bulk }}\right)$ of argon confined between hard walls and subject to the wall potential model of Werder et al. [18] up to a distance of a cutoff from the boundary. By reducing the temperature, while increasing the density, the amplitude of the oscillations becomes higher. $-+-\left(T^{*}=1.8, \rho^{*}=0.60\right)$, $-\times-\left(T^{*}=1.1, \rho^{*}=0.81\right)$, and $-*-\left(T^{*}=0.7, \rho^{*}=0.89\right)$.

cutoff radius $\left(r_{c}\right)$ of $1.0 \mathrm{~nm}$. We note that increasing the cutoff from $1 \mathrm{~nm}$ to $2 \mathrm{~nm}$ does not affect the quality of the results. The equations of motion are integrated using the leap frog scheme with a time step of $10 \mathrm{fs}$. We perform the MD simulations at different state points of the fluid and report quantities in reduced units $\left(T^{*}=k_{B} T / \epsilon, \rho^{*}=\rho \sigma^{3}\right.$, and $P^{*}$ $\left.=P \sigma^{3} / \epsilon\right)$ where $\tau=\left(\frac{m \sigma^{2}}{\epsilon}\right)^{0.5}$ is the characteristic time of the Lennard-Jones potential. In hybrid algorithms, the elimination of periodic boundary conditions in the atomistic domain hinders the maintenance of a uniform density across the domain and the proper calculation of the virial pressure. In order to correct for the "missing component" of the virial pressure, a boundary force is applied in the atomistic domain $[13,14,17]$. Hence, we impose NPBCs in the $x$ direction with a wall force $F_{m}$ to exert the correct mean virial pressure $\left(P_{U}\right)$ on the MD system along with a specular wall to impose the ideal kinetic part $\left(P_{K}\right)$ of the system pressure:

$$
P=P_{K}+P_{U}=k_{B} T \rho_{n}+\rho_{n} \int_{0}^{r_{c}} F_{m}(r) d r,
$$

where $k_{B}$ is the Boltzmann constant.

In Ref. [18] it was proposed to compute the wall force $\left(F_{m}\right)$ from the pair potential [Eq. (1)] and the pair correlation function $[g(r)]$ of the working fluid. This technique was shown to alleviate many of the drawbacks of existing methods, and it constitutes the basis of the present algorithm. Thus the Lennard-Jones force of each particle weighted by $g(r)$ is integrated over the part of the cutoff sphere that lies outside of the atomistic domain (cf. Fig. 1),

$$
F_{m}\left(r_{w}\right)=-2 \pi \rho_{n} \int_{z=r_{w}}^{r_{c}} \int_{x=0}^{\sqrt{r_{c}^{2}-z^{2}}} g(r) \frac{\partial U_{12-6}(r)}{\partial r} \frac{z}{r} x d x d z .
$$

At the supercritical state point $\left(T^{*}=1.8, \rho^{*}=0.6\right)$ [22] this approach was found to reduce drastically the spurious density fluctuations [18]. 


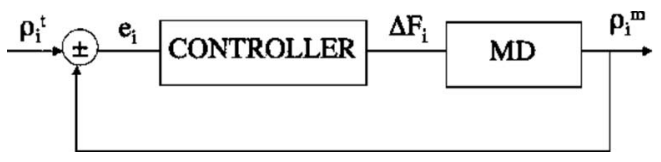

FIG. 3. Schematic of the control algorithm for reducing density fluctuations.

We examine the validity of this method in two additional state points in the liquid regime: namely, $\left(T^{*}=1.1, \rho^{*}=0.81\right)$ and $\left(T^{*}=0.7, \rho^{*}=0.89\right)$. The size of the computational domain is $5 \mathrm{~nm} \times 5 \mathrm{~nm} \times 5 \mathrm{~nm}$. The periodicity is broken in the $x$ direction. The system is weakly coupled to a Berendsen thermostat [23] with a time constant of $0.1 \mathrm{ps}$. The heat bath is imposed cellwise using $5 \times 5 \times 1$ cells. After equilibration we heat only the atoms located in the cells close to the $x$ boundary. For these two new state points the method is found to encounter difficulties when lowering the temperature, while increasing the density, at constant pressure, leading to density oscillations close to the boundary (Fig. 2). The amplitude of these oscillations amounts to $15 \%$ and is well below previously reported values in hybrid simulations [18] but they may still cause unnecessary disturbances to the atomistic system. We attribute these persistent oscillations to the deterministic boundary force [Eq. (2)] and lack of fluctuations inherent to the atomistic system.

In order to reduce these spurious oscillations we develop a control algorithm for the mean external boundary force applied to the MD system. The control approach is sketched in Fig. 3. Each iteration involves the following steps: we start by applying the external boundary force as proposed in Eq. (2). Then we measure the density in short time intervals filtering away high-frequency noise. This filtering makes it more suitable for coupling the atomistic to the continuum description, and it is found to improve the convergence of the method. The density $\rho^{m^{\prime}}$ is measured with a spatial resolution $\delta x$ of $0.0166 \mathrm{~nm}$ in time intervals of $30 \mathrm{ps}$ and processed twice through a Gaussian filter resulting in the filtered $\rho^{m}$ quantity

$$
\begin{aligned}
& \rho^{m^{\prime \prime}}(x)=\frac{1}{\epsilon} \int \rho^{m^{\prime}}(x) \exp \left(-\frac{(x-y)^{2}}{\epsilon^{2}}\right) d y, \\
& \rho^{m}(x)=\frac{1}{\epsilon} \int \rho^{m^{\prime \prime}}(x) \exp \left(-\frac{(x-y)^{2}}{\epsilon^{2}}\right) d y,
\end{aligned}
$$

where $\epsilon=2 \delta x$. The cutoff used for the discrete evaluation of the convolution is $3 \delta x$ and the filter obtains the following discrete form:

$$
\rho_{i}^{m^{\prime \prime}}= \begin{cases}\rho_{i}^{m^{\prime}} & \text { for } i=1 \text { or } i=N, \\ 0.3045 \rho_{i \pm 1}^{m^{\prime}}+0.3910 \rho_{i}^{m^{\prime}} & \text { for } i=2 \text { or } i=N-1, \\ 0.1117 \rho_{i \pm 2}^{m^{\prime}}+0.2365 \rho_{i \pm 1}^{m^{\prime}}+0.3036 \rho_{i}^{m^{\prime}} & \text { for } i=3 \text { or } i=N-2, \\ 0.0301 \rho_{i \pm 3}^{m^{\prime}}+0.1050 \rho_{i \pm 2}^{m^{\prime}}+0.2223 \rho_{i \pm 1}^{m^{\prime}}+0.2854 \rho_{i}^{m^{\prime}} & \text { for } 4 \leq i \leq N-3,\end{cases}
$$

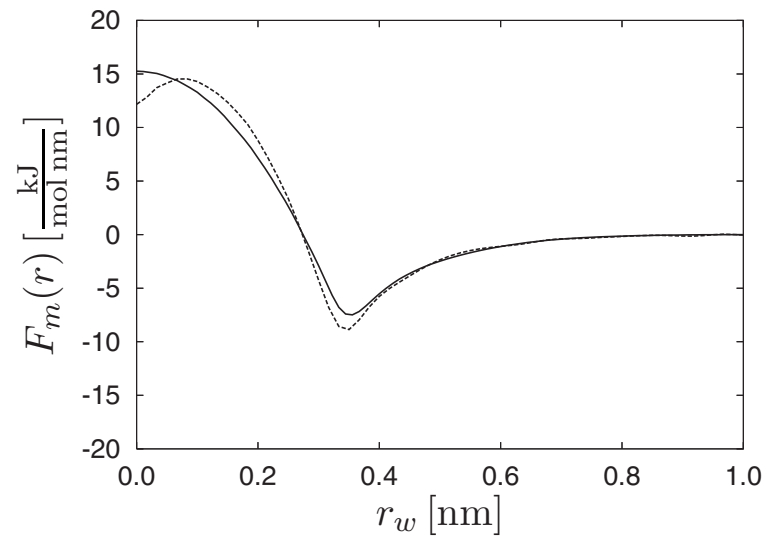

(a)

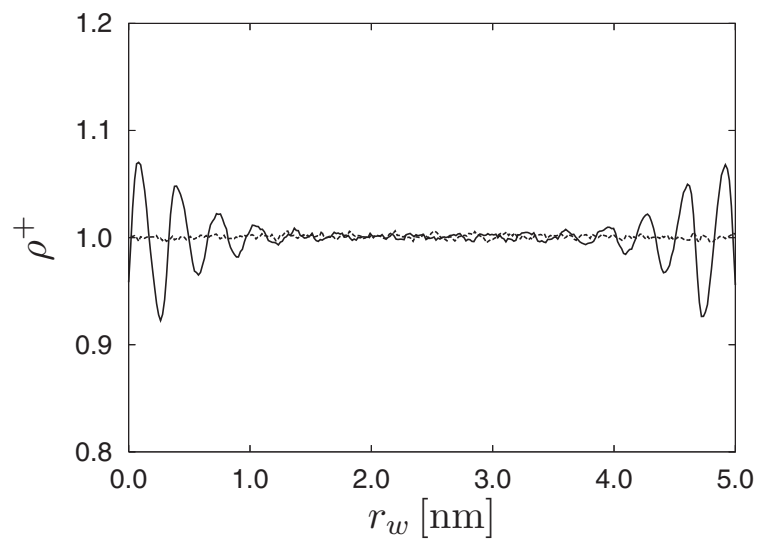

FIG. 4. (a) The initial external boundary force (solid line) computed taking the fluid structure into account and the resulting one (dashed line) after applying the control algorithm at the state point $\left(T^{*}=1.1, \rho^{*}=0.81\right)$. (b) The corresponding uncontrolled (solid line) and controlled reduced density values (dashed line). The value used for $K_{P}$ is $0.083 \frac{\mathrm{nm} 3 \mathrm{~kJ}}{\mathrm{amu} \mathrm{mol}}$, and both the force and density have been sampled over $10 \mathrm{~ns}$. 

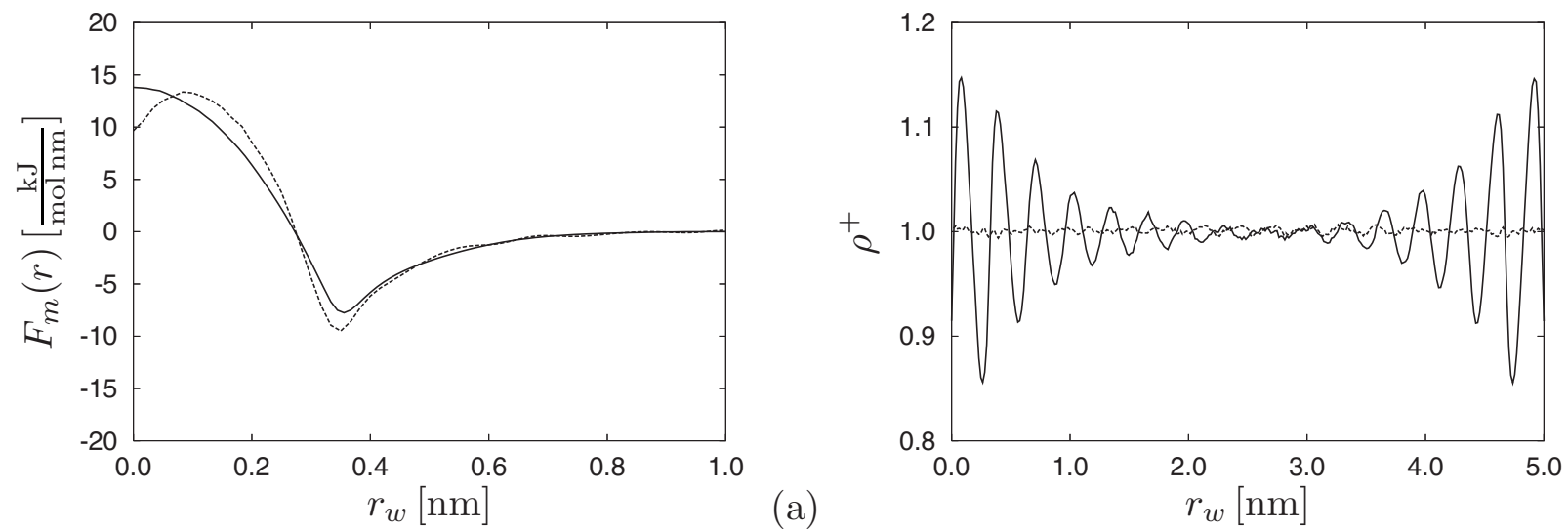

FIG. 5. (a) The initial external boundary force (solid line) computed taking the fluid structure into account and the resulting one (dashed line) after applying dynamic control theory at the state point $\left(T^{*}=0.7, \rho^{*}=0.89\right)$. (b) The corresponding uncontrolled (solid line) and controlled reduced density values (dashed line). The value used for $K_{P}$ is $0.0830 \frac{\mathrm{nm}^{3} \mathrm{~kJ}}{\mathrm{amu} \mathrm{mol}}$, and both the force and density have been sampled over $10 \mathrm{~ns}$.

where $N$ is the number of the measured density values. We then evaluate the error as

$$
e\left(r_{w}\right)=\rho^{t}-\rho^{m}\left(r_{w}\right)
$$

where $r_{w}$ is the distance to the boundary, $\rho^{t}$ the desired constant target density, and $\rho^{m}$ the measured filtered value. We compute the gradient of this error as $\epsilon\left(r_{w}\right)=\nabla e\left(r_{w}\right)=$ $-\nabla \rho^{m}\left(r_{w}\right)$ and amplify this with a factor $K_{P}$ to obtain the changes $\Delta F$ in the boundary force as

$$
\Delta F_{i}=K_{P} \epsilon_{i},
$$

for each $i$ th bin. The boundary force is finally computed as

$$
F_{i}^{\text {new }}=F_{i}^{\text {old }}+\Delta F_{i}
$$

After the root mean square $(E)$ of the error reaches a value less than a prescribed value, here $1 \%$, we consider that the method has converged and we can start measuring the density for assessing the quality of the result. The controller online keeps acting on the system. $E$ is computed in time intervals of 300 ps.

We test this approach for the state points $\left(T^{*}=1.1, \rho^{*}\right.$ $=0.81)$ and $\left(T^{*}=0.7, \rho^{*}=0.89\right)$, where the mean force algorithm of Werder et al. [18] failed to fully eliminate the fluctuations. We set $K_{P}=0.0830 \frac{\mathrm{nm}^{3} \mathrm{~kJ}}{\mathrm{amu} \mathrm{mol}}$, and the results shown in Figs. 4 and 5 demonstrate that in both cases the method converges and eliminates the density oscillations. When compared with the initial force, we observe a decrease of the magnitude of the force close to the boundary and a shift for the location of the minimum. At larger distances from the wall $\left(r_{w}>0.6 \mathrm{~nm}\right)$ the shape of the force is not significantly altered. In Fig. 6 we compare the convergence of the method for the values of $K_{P}=0.0830 \frac{\mathrm{nm}^{3} \mathrm{~kJ}}{\mathrm{amu} \mathrm{mol}}$ and $K_{P}=0.0166 \frac{\mathrm{nm}^{3} \mathrm{~kJ}}{\mathrm{amu} \mathrm{mol}}$. We find a value of $K_{P}=0.0830 \frac{\mathrm{nm}^{3} \mathrm{~kJ}}{\mathrm{amu} \mathrm{mol}}$ to guarantee good stability properties and fast convergence. In the cases considered the method has converged approximately after $550 \tau$ at the state point $\left(T^{*}=1.1, \rho^{*}=0.81\right)$ and after $800 \tau$ at the state point $\left(T^{*}=0.7, \rho^{*}=0.89\right)$.

\section{VALIDATION IN DIFFERENT FLOW CONFIGURATIONS}

In addition, we examine the performance of the control algorithm in the case of parallel flow at the state point $\left(T^{*}\right.$ $\left.=0.7, \rho^{*}=0.89\right)$. The periodicity is broken in the flow direction $(x)$. The system is weakly coupled to a Berendsen thermostat [23] with a time constant of $0.1 \mathrm{ps}$. The heat bath is imposed cellwise using $5 \times 5 \times 1$ cells. The flow is imposed by adjusting the mean flow velocity of the atoms [24] in the computational cells with center points $(x=0.5)$ and $(x=4.5)$ to achieve a mean velocity of $25 \mathrm{~m} \mathrm{~s}^{-1}$. After equilibration we heat only the atoms located in the boundary boxes at the inlet and the outlet. As described in [18] atoms at the nonperiodic boundary of the computational domain bounce with hard walls, which move with the local fluid velocity. At the end of each time step these walls are reset to their initial

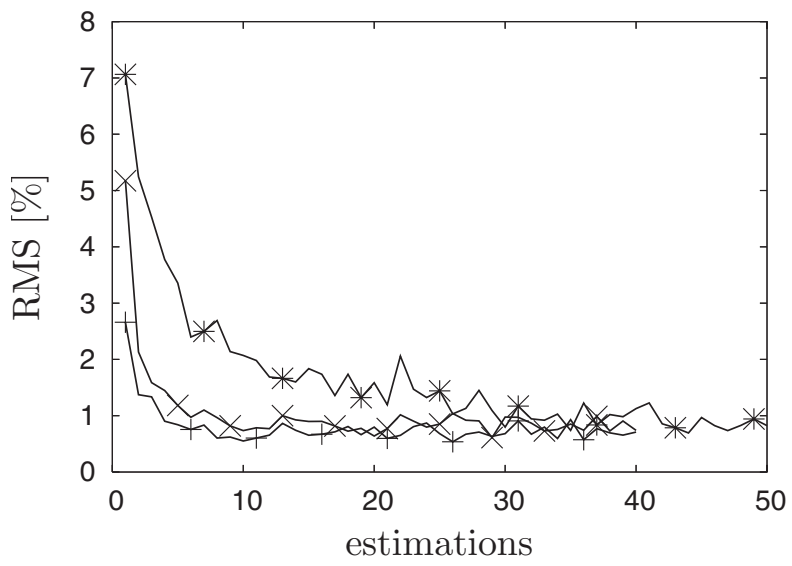

FIG. 6. Convergence of the root mean square (rms) of the error $\left(E=\sqrt{\frac{1}{N} \sum_{i=1}^{N} e_{i}^{2}}\right)$ in density when adjusting the external boundary force: $-+-\left(T^{*}=1.1, \rho^{*}=0.81, K_{P}=0.0830 \frac{\mathrm{nm}^{3} \mathrm{~kJ}}{\mathrm{amu} \mathrm{mol}}\right), C^{*}-\left(T^{*}\right.$ $\left.=0.7, \rho^{*}=0.89, K_{P}=0.0166 \frac{\mathrm{nm}^{3} \mathrm{~kJ}}{\mathrm{amu} \mathrm{mol}}\right)$, and $-\times-\left(T^{*}=0.7, \rho^{*}=0.89\right.$, $\left.K_{P}=0.0830 \frac{\mathrm{nm}^{3} \mathrm{~kJ}}{\mathrm{amu} \mathrm{mol}}\right)$. Each estimation of the rms corresponds to ten updates of the online control force. 

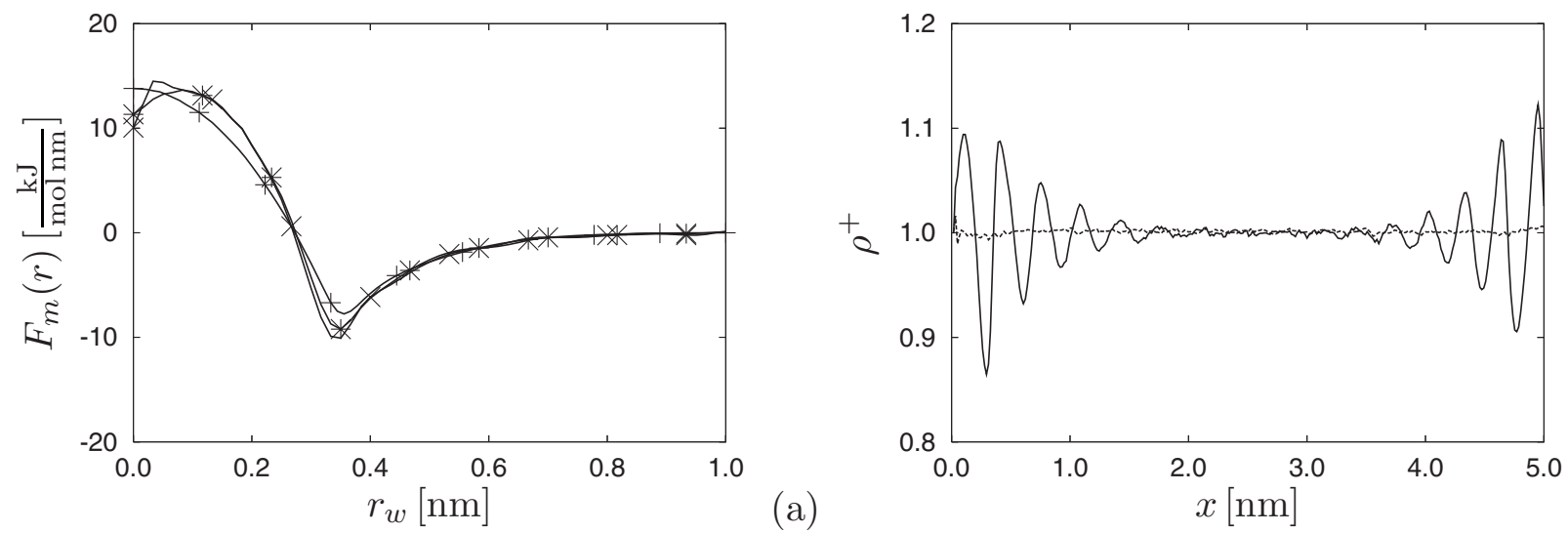

FIG. 7. (a) The external boundary forces at the state point $\left(T^{*}=0.7, \rho^{*}=0.89\right)$ in the case of parallel flow: $(-+-)$ uncontrolled case, $(-$ $\times-)$ controlled case (inflow), (_*-) controlled case (outflow). (b) The resulting reduced density $\left(\rho^{+}\right)$profiles in the $x$ direction: (solid line) uncontrolled case, (dashed line) controlled case. The value used for $K_{P}$ is $0.083 \frac{\mathrm{nm}^{3} \mathrm{~kJ}}{\mathrm{amu} \text { mol }}$, and both the force and density have been sampled over $10 \mathrm{~ns}$.

positions to maintain a fixed frame of reference. As a consequence some particles may remain outside the computational domain and are reinserted in regions of inflow. Their final position after reinsertion is determined using the Usher algorithm [25]. This removal and insertion of particles is not symmetric, and therefore we control the forces at the inlet and outlet of the computational domain separately with a $K_{P}$ of $0.0830 \frac{\mathrm{nm}^{3} \mathrm{~kJ}}{\mathrm{amu} \mathrm{mol}}$. We update the control force every $60 \mathrm{ps}$. In Fig. 7 we show the external boundary force in the controlled and uncontrolled cases and the resulting reduced density profiles. The perturbed density, with an amplitude up to $11 \%$ in the uncontrolled case, also leads to oscillations in the streaming velocity $(u)$ of the atoms (cf. Fig. 8). The controller successfully eliminates the deviations from the target value for both quantities at both the inlet and outlet boundaries.

Finally, we validate the control algorithm for the case of Couette flow of liquid argon $\left(T^{*}=1.1, \rho^{*}=0.81\right)$ confined between two graphite surfaces. A sketch of the flow geometry is shown in Fig. 9. The size of the computational domain is $30.0 \mathrm{~nm} \times 4.3 \mathrm{~nm} \times 4.9 \mathrm{~nm}$, small enough to allow a fully

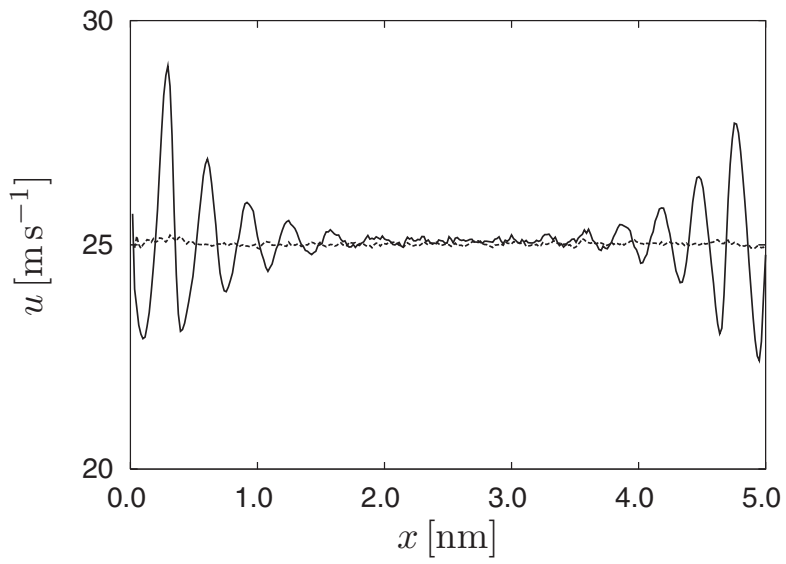

FIG. 8. Resulting velocity profiles (sampled over $10 \mathrm{~ns}$ ) in the $x$ direction at the state point $\left(T^{*}=0.7, \rho^{*}=0.89\right)$ in the case of parallel flow: (solid line) uncontrolled case, (dashed line) controlled case. atomistic simulation which we will use as a reference. The number of computational boxes used for heating is $30 \times 1$ $\times 1$. The same resolution is used to sample the velocities that serve as a boundary condition for the continuum solver. The flow is imposed by moving the upper wall with a velocity $v=100 \mathrm{~m} \mathrm{~s}^{-1}$.

In the hybrid approach we apply the Schwarz alternating method with an overlap region of four cells $(4 \mathrm{~nm})$. After equilibration only the $x$ and $z$ components of the particle velocities are weakly coupled to the thermostat. Details about the exchange of boundary conditions between the MD and the continuum region, described by incompressible Navier-Stokes (NS) equations, can be found in [18]. In the present case the solution to the NS equations is a linear streamwise velocity profile. In the hybrid approach the system contains a graphite surface and the argon atoms form layers in its vicinity. During equilibration we dynamically insert and remove particles in order to match the state point of the pure system. The upper and lower MD region in the hybrid case have the dimensions $10.0 \mathrm{~nm} \times 4.3 \mathrm{~nm}$

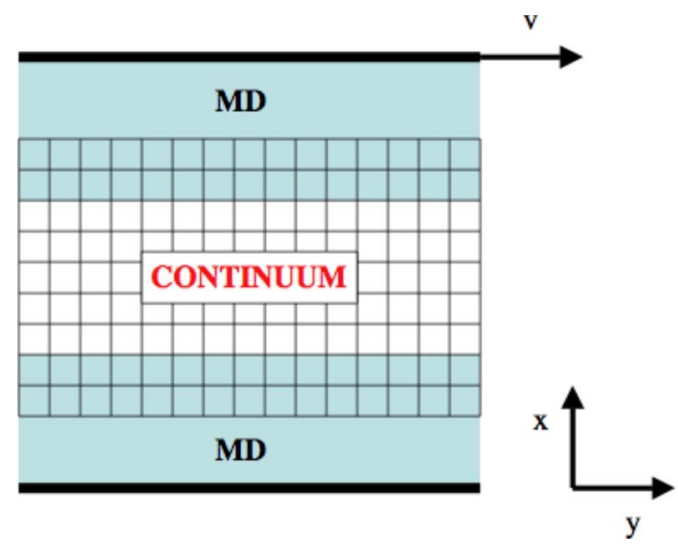

FIG. 9. (Color online) Sketch of the hybrid simulation for the Couette flow. Shaded regions denote the domain of MD simulations and grid cells indicate simulations using the Navier-Stokes equations. 

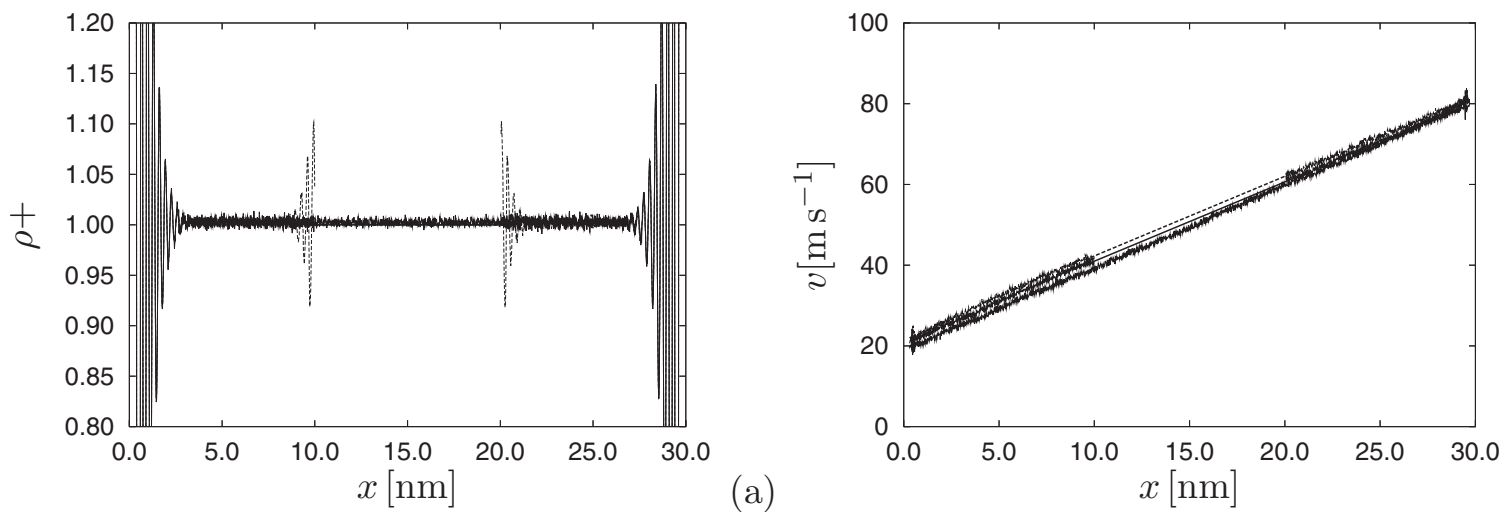

FIG. 10. (a) Reduced density profiles in the Couette flow of the reference MD simulations and of the hybrid controlled (solid line) and uncontrolled (dashed line) cases. Density oscillations of the order of $10 \%$ in the uncontrolled case are eliminated by the controller. (b) Resulting velocity profiles in the Couette flow of the reference MD simulations and the hybrid controlled (solid line) and uncontrolled (dashed line) cases.

$\times 4.9 \mathrm{~nm}$ (ten boxes in the $x$ and one box in the $y$ and $z$ directions), large enough to resolve the extensive physical perturbations in the fluid density at the fluid-solid interface.

In a cycle of the hybrid algorithm, we impose the boundary condition from the continuum to the MD, equilibrate the MD system for 20 ps to reach a new quasisteady state, and subsequently sample the velocities for $100 \mathrm{ps}$ to extract the $\mathrm{BC}$ for the continuum. The flow reaches a steady state after $10 \mathrm{~ns}$, and we sample the results from $10 \mathrm{~ns}$ to $35 \mathrm{~ns}$ every 1 ps with a total of 25000 samples. In Fig. 10 we show the velocity and density profiles obtained from the hybrid simulations. We observe large physical perturbations in the density at the graphite surface and spurious oscillations at the continuum-MD interface in the uncontrolled case which are eliminated in the controlled one. The velocity profiles are similar in both cases.

\section{CONCLUSIONS}

In summary, we have presented a control algorithm to eliminate density fluctuations in the coupling of atomistic models with continuum descriptions of dense liquids using Schwarz domain decomposition. A dynamic controller, based on the errors measured in the local fluid density, enhances a current state-of-the-art [18] boundary forcing. The algorithm is validated for fluids at rest, and it is shown to eliminate density oscillations with amplitude of the order of $15 \%$. In simulations of a uniform parallel flow the controller prevents the propagation of the perturbations, removing density and velocity oscillations of $11 \%$. Finally we demonstrated the capability of the algorithm for multiscale simulations by successfully coupling of MD to a continuum description for the Couette flow problem.

The present work in our group is concerned with the extension of the proposed scheme to coupling continuum models (a recent extension involves lattice-Boltzman [26]) mesoscale descriptions (e.g., coarse-grained MD [27,28]) with atomistic simulations of polyatomic liquids such as water. We are implementing the reinsertion algorithm for water proposed in [29], and ongoing work involves the determination of the external boundary force. In addition, this control algorithm may be applied to pure MD simulations of water in order to avoid periodic boundary conditions [30], while another possible extension of this approach is that the boundaries separating the atomistic to continuum region could be irregular (such as spherical) depending on the geometry of the problem. We note that the simplicity of the present algorithm can be adopted for a number of existing atomisticcontinuum coupling techniques, and we believe that the proposed control methodology will help facilitate the development of further advanced multiscale simulations.
[1] R. J. Chen, S. Bangsaruntip, K. A. Drouvalakis, N. W. S. Kam, M. Shim, Y. M. Li, W. Kim, P. J. Utz, and H. J. Dai, Proc. Natl. Acad. Sci. U.S.A. 100, 4984 (2003).

[2] J. Li, H. T. Ng, A. Cassell, W. Fan, H. Chen, Q. Ye, J. Koehne, J. Han, and M. Meyyappan, Nano Lett. 3, 597 (2003).

[3] Y. Lin, S. Taylor, H. Li, K. A. Shiral Fernando, L. Qu, W. Wang, L. Gu, B. Zhou, and Y. Sun, J. Mater. Chem. 14, 527 (2004).

[4] M. Zheng, A. Jagota, E. D. Semke, B. A. Diner, R. S. McLean,
S. R. Lustig, R. E. Richardson, and N. G. Tassi, Nat. Mater. 2, 338 (2003).

[5] K. Watanabe, T. Takayama, S. Ogata, and S. Isozaki, AIChE J. 49, 1956 (2003).

[6] N. G. Hadjiconstantinou, J. Comput. Phys. 154, 245 (1999).

[7] X. Nie, M. O. Robbins, and S. Chen, Phys. Rev. Lett. 96, 134501 (2006).

[8] C. Cottin-Bizonne, J. Barrat, L. Bocquet, and E. Charlaix, Nat. Mater. 2, 237 (2003). 
[9] Peter A. Thompson and Mark O. Robbins, Phys. Rev. A 41, 6830 (1990).

[10] H. Brenner and V. Ganesan, Phys. Rev. E 61, 6879 (2000).

[11] R. Delgado-Buscalioni and P. V. Coveney, Phys. Rev. E, 67, 046704 (2003).

[12] P. Koumoutsakos, Annu. Rev. Fluid Mech. 37, 457 (2005).

[13] S. T. O'Connell and P. A. Thompson, Phys. Rev. E 52, R5792 (1995).

[14] E. G. Flekkøy, G. Wagner, and J. Feder, Europhys. Lett. 52, 271 (2000).

[15] E. G. Flekkoy, R. Delgado-Buscalioni, and P. V. Coveney, Phys. Rev. E 72, 026703 (2005).

[16] G. De Fabriitis, R. Delgado-Buscalioni, and P. V. Coveney, Phys. Rev. Lett. 97, 134501 (2006).

[17] X. B. Nie, S. Y. Chen, W. N. E, and M. O. Robbins, J. Fluid Mech. 500, 55 (2004).

[18] T. Werder, J. H. Walther, and P. Koumoutsakos, J. Comput. Phys. 205, 373 (2005).

[19] I. V. Pivkin and G. E. Karniadakis, Phys. Rev. Lett. 96, 206001 (2006).

[20] N. G. Hadjiconstantinou, A. L. Garcia, M. Z. Bazant, and G.
He, J. Comput. Phys. 187, 274 (2003)

[21] N. G. Hadjiconstantinou, Bull. Pol. Acad. Sci.: Tech. Sci. 53, 335 (2005).

[22] J. K. Johnson, J. A. Zollweg, and K. E. Gubbins, Mol. Phys. 78, 591 (1993).

[23] H. J. C. Berendsen, J. P. M. Postma, W. F. van Gunsteren, A. DiNola, and J. R. Haak, J. Chem. Phys. 81, 3684 (1984).

[24] J. H. Walther, T. Werder, R. L. Jaffe, and P. Koumoutsakos, Phys. Rev. E 69, 062201 (2004).

[25] R. Delgado-Buscalioni and P. V. Coveney, Philos. Trans. R. Soc. London, Ser. A 362, 1639 (2004).

[26] A. Dupuis, E. M. Kotsalis, and P. Koumoutsakos, Phys. Rev. E 75, 046704 (2007).

[27] M. Praprotnik, L. Delle Site, and K. Kremer, J. Chem. Phys. 123, 224106 (2005).

[28] M. Praprotnik, L. Delle Site, and K. Kremer, Phys. Rev. E 73, 066701 (2006).

[29] G. De Fabriitis, R. Delgado-Buscalioni, and P. V. Coveney, J. Chem. Phys. 121, 12139 (2004).

[30] W. Im, S. Bernechè, and B. Roux, J. Chem. Phys. 114, 2924 (2001). 